\title{
Does Gender Affect the Outcomes of Multiple Valve Heart Surgery?
}

\section{Iskander Al-Githmi}

Department of Surgery, Division of Cardiothoracic Surgery, King Abdulaziz University Hospital, Jeddah, Saudi Arabia.

Email: algithmi@hotmail.com

Received December $17^{\text {th }}, 2011$; revised January $26^{\text {th }}, 2012$; accepted February $4^{\text {th }}, 2012$

\begin{abstract}
Background: Multiple valve surgery exposes patients to major morbidity and mortality. Little is known about the effect of gender on the outcomes of multiple valve surgery. Methods: In 69 patients who had multiple valve surgery for rheumatic valvular heart disease, 51 patients had mitral and aortic valve replacement, 9 patients had mitral and aortic valve replacement and tricuspid valve repair, 4 patients had mitral valve replacement and tricuspid valve repair, 4 patients had mitral and tricuspid valve repair, and 1 patient had mitral and tricuspid valve replacement. Outcomes were evaluated with univariate analysis. Results: Women had significantly smaller body surface area and smaller left ventricular end-systolic area than men. Women and men had similar left ventricular ejection fraction and New York Heart Association functional class. Univariate analysis showed that in women (but not men), older age, atrial fibrillation, lower left ventricular ejection fraction, and New York Heart Association functional class II and III were associated with longer hospital and intensive care unit stay. In men (but not women), longer cardiopulmonary bypass time was associated with increased left ventricular end-systolic area at 12 months after surgery. Longer aortic cross-clamp time was associated with increased left ventricular end-systolic area in men but only weakly in women. Conclusion: We concluded that gender is an independent predictor of outcomes after multiple valve heart surgery.
\end{abstract}

Keywords: Gender; Multiple Valves; Surgery; Outcomes

\section{Introduction}

During the past 2 decades, improvements have occurred in the clinical outcomes of patients with multiple valvular heart disease. Many studies have examined the effect of gender-related differences and outcomes after isolated valvular heart surgery, isolated coronary artery bypass grafting, and valvular heart surgery with coronary artery bypass grafting [1-10]. However, knowledge about the effects of gender-related differences on the outcomes of multiple valvular heart surgery is limited. The current prospective study aimed to examine the association of gender with the outcomes of multiple valvular heart surgery.

\section{Materials and Methods}

Multiple valvular heart surgery was performed on 73 consecutive patients from January 1999 to December 2005. Of these 73 patients, 4 women were excluded because of missing data, leaving 69 patients (43 women and 26 men) who were enrolled in the study and were prospectively followed. All 69 patients had rheumatic valvular heart disease and underwent primary multiple valve surgery, defined as a single operation that involved $>1$ valve that included a combination of aortic valve replacement, mitral valve repair or replacement, and/or tricuspid valve repair or replacement (Table 1).

The outcomes reviewed included duration of intensive care unit (ICU) stay, hospital stay, and mechanical ventilation; and postoperative atrial fibrillation, left ventricular ejection fraction, left ventricular end-systolic area at 12 months after surgery and mortality at 30 days after surgery.

\section{Data Analysis}

Two independent samples t-test, Mann-Whitney test, Kruskal-Wallis test, exact chi-square test, and Spearman's correlation coefficient were used to assess the demographic characteristics of the patients and the effects of various risk factors on the different outcomes of men and women separately, wherever appropriate. All the statistical tests were performed using SPSS for Windows (Release 14.0; SPSS Inc., Chicago, Illinois, USA). The level of significance was set at $\mathrm{P} \leq 0.05$.

\section{Results}

Women and men were similar in age, left ventricular 
ejection fraction, New York Heart Association functional class, medical comorbidities, valve disease type, and cardiopulmonary bypass and cross-clamp times (Table 1). Women had significantly smaller body surface area and smaller left ventricular end-systolic area than men (Table 1). Surgery performed in most patients was combined mitral and aortic valve replacement (Table 1).

Univariate analysis showed that in women (but not men), older age, atrial fibrillation, lower left ventricular ejection fraction, and New York Heart Association functional class II and III were associated with longer hospital and ICU stay (Table 2). In men (but not women), longer cardiopulmonary bypass time was associated with increased left ventricular end-systolic area at 12 months after surgery (Table 2). In addition, longer aortic crossclamp time was associated with increased left ventricular end-systolic area in men but only weakly in women (Table 2).

Table 1. Clinical characteristics of patients who had multiple valve heart surgery*.

\begin{tabular}{|c|c|c|c|}
\hline Clinical characteristics & Women & Men & $P \leqslant \dagger$ \\
\hline Number of patients & 43 & 26 & - \\
\hline Age (y) & $50 \pm 11$ & $49 \pm 15$ & NS \\
\hline Body surface area $\left(\mathrm{m}^{2}\right)$ & $1.5 \pm 0.1$ & $1.7 \pm 0.2$ & 0.003 \\
\hline Left ventricular end-systolic area $\left(\mathrm{mm}^{2}\right)$ & $36(29,31)$ & $39(35,49)$ & 0.02 \\
\hline \multicolumn{4}{|l|}{ Left ventricular ejection fraction (\%) } \\
\hline$<40$ & $3(7 \%)$ & $2(8 \%)$ & \\
\hline $40-60$ & $20(47 \%)$ & $14(56 \%)$ & NS \\
\hline$>60$ & $20(47 \%)$ & $9(36 \%)$ & \\
\hline \multicolumn{4}{|l|}{ NYHA class } \\
\hline I & $6(4 \%)$ & $3(12 \%)$ & \\
\hline II & $23(53 \%)$ & $13(50 \%)$ & NS \\
\hline III & $14(32 \%)$ & $9(35 \%)$ & \\
\hline IV & 0 & $1(4 \%)$ & \\
\hline \multicolumn{4}{|l|}{ Comorbidities } \\
\hline Atrial fibrillation & $28(68 \%)$ & $15(63 \%)$ & NS \\
\hline Congestive heart failure & $7(18 \%)$ & $9(35 \%)$ & NS \\
\hline Hypertension & $4(10 \%)$ & $2(8 \%)$ & NS \\
\hline Diabetes mellitus & $7(16 \%)$ & $0(0 \%)$ & NS \\
\hline Stroke & $2(5 \%)$ & $1(4 \%)$ & NS \\
\hline COPD & $2(8 \%)$ & $0(0 \%)$ & NS \\
\hline \multicolumn{4}{|l|}{ Aortic valve disease } \\
\hline Stenosis & $26(61 \%)$ & $12(46 \%)$ & NS \\
\hline Regurgitation & $35(81 \%)$ & $25(96 \%)$ & NS \\
\hline Mixed & $23(54 \%)$ & $12(46 \%)$ & NS \\
\hline \multicolumn{4}{|l|}{ Mitral valve disease } \\
\hline Stenosis & $32(74 \%)$ & $18(69 \%)$ & NS \\
\hline Regurgitation & $36(84 \%)$ & $21(81 \%)$ & NS \\
\hline Mixed & $25(58 \%)$ & $13(50 \%)$ & NS \\
\hline \multicolumn{4}{|l|}{ Tricuspid valve disease } \\
\hline Stenosis & $0(0 \%)$ & $1(4 \%)$ & NS \\
\hline Regurgitation & $31(72 \%)$ & $18(69 \%)$ & NS \\
\hline \multicolumn{4}{|l|}{ Operations } \\
\hline $\mathrm{MVR}+\mathrm{AVR}$ & $29(67 \%)$ & $22(85 \%)$ & \\
\hline $\mathrm{MVR}+\mathrm{AVR}+\mathrm{TV}$ repair & $6(14 \%)$ & $3(12 \%)$ & \\
\hline MVR + TV repair & $3(7 \%)$ & $1(4 \%)$ & NS \\
\hline MV repair + TV repair & $4(9 \%)$ & $0(0 \%)$ & \\
\hline $\mathrm{MVR}+\mathrm{TVR}$ & $1(2 \%)$ & $0(0 \%)$ & \\
\hline Cardiopulmonary bypass time (min) & $142(120,160)$ & $153(125,188)$ & NS \\
\hline Aortic cross-clamp time (min) & $119(95,129)$ & $121(106,147)$ & NS \\
\hline Postoperative mechanical ventilation $(>1 \mathrm{~d})$ & $58(43,63)$ & $48(22,55)$ & NS \\
\hline
\end{tabular}

${ }^{*} \mathrm{~N}=69$ patients. Data reported as mean $\pm \mathrm{SD}$; number (percent) patients, or median (interquartile range). Abbreviations: AVR, aortic valve replacement; COPD, chronic obstructive pulmonary disease; MV, mitral valve; MVR, mitral valve replacement; NYHA, New York Heart Association; TV, tricuspid valve; TVR, tricuspid valve replacement; $\uparrow$ NS, not significant $(\mathrm{P}>0.05)$. 
Table 2. Univariate analysis of postoperative outcomes after multiple valve heart surgery ${ }^{*}$.

\begin{tabular}{lllll}
\hline Risk Factor & $\begin{array}{l}\text { Length of } \\
\text { hospital stay }\end{array}$ & $\begin{array}{l}\text { Intensive care } \\
\text { unit stay } \\
>1 \text { day }\end{array}$ & $\begin{array}{l}\text { Mechanical } \\
\text { ventilation } \\
>1 \text { day }\end{array}$ & $\begin{array}{l}\text { Left ventricular } \\
\text { end-systolic area at } 12 \\
\text { months after surgery }\end{array}$ \\
\hline Age & & & & \\
Women & $0.418^{\# *}$ & $58(34,63)$ & $58(38,63)$ & 0.169 \\
Men & -0.046 & $48(22,55)$ & $48(22,55)$ & -0.496 \\
All & $0.25^{*}$ & $52(22,63)$ & $52(22,63)$ & -0.137
\end{tabular}

Left ventricular ejection fraction (\%)

\begin{tabular}{lcllll} 
Women & $<40$ & $12(8,35)$ & $2(67 \%)$ & $0(0 \%)$ & $32(32,32)$ \\
& $40-60$ & $10(6,36)$ & $3(15 \%)$ & $3(15 \%)$ & $31(25,45)$ \\
\multirow{3}{*}{ Men } & $>60$ & $12(7,25)$ & $0(0 \%)$ & $0(0 \%)$ & $32(15,45)$ \\
& $<40$ & $9(8,9)$ & $0(0 \%)$ & $0(0 \%)$ & $0(0)$ \\
\multirow{4}{*}{ All } & $40-60$ & $12(7,27)$ & $1(7 \%)$ & $1(7 \%)$ & $42(39,49)$ \\
& $>60$ & $13(8,30)$ & $1(11 \%)$ & $1(11 \%)$ & $35(28,39)$ \\
& $<40$ & $9(8,35)$ & $2(40 \%)$ & $0(0 \%)$ & $32(32,32)$ \\
& $40-60$ & $10(6,36)$ & $4(12 \%)$ & $4(12 \%)$ & $36(25,49)$ \\
& $>60$ & $12(7,30)$ & $1(3 \%)$ & $1(3 \%)$ & $33(15,45)$
\end{tabular}

Atrial fibrillation

\begin{tabular}{llllll} 
Women & Yes & $12(8,36)$ & $5(18 \%)$ & $3(11 \%)$ & $33(15,45)$ \\
\multirow{3}{*}{ Men } & No & $8(6,20)$ & $0(0 \%)$ & $0(0 \%)$ & $28(25,33)$ \\
& Yes & $12(8,30)$ & $1(6 \%)$ & $1(7 \%)$ & $37(30,43)$ \\
\multirow{2}{*}{ All } & No & $12(9,27)$ & $1(11 \%)$ & $1(11 \%)$ & $37(28,41)$ \\
& Yes & $12(8,36)$ & $6(14 \%)$ & $4(9 \%)$ & $35(15,45)$ \\
& No & $10(6,27)$ & $1(5 \%)$ & $1(5 \%)$ & $29(25,41)$
\end{tabular}

Chronic obstructive pulmonary disease

\begin{tabular}{llllll} 
Women & Yes & $13(8,18)$ & $2(100 \%)$ & $2(100 \%)$ & $0(0)$ \\
\multirow{3}{*}{ Men } & No & $10(6,36)$ & $2(9 \%)$ & $1(5 \%)$ & $32(25,45)$ \\
& Yes & & $0(0 \%)$ & $0(0 \%)$ & $0(0)$ \\
\multirow{2}{*}{ All } & No & $12(7,30)$ & $3(20 \%)$ & $3(20 \%)$ & $41(39,49)$ \\
& Yes & $13(8,18)$ & $2(100 \%)$ & $2(100 \%)$ & $0(0)$ \\
& No & $11(6,36)$ & $5(14 \%)$ & $4(11 \%)$ & $33(25,49)$
\end{tabular}

Cardiopulmonary bypass time

$\begin{array}{lllll}\text { Women } & 0.247 & 147(81,304) & 147(81,304) & -0.068 \\ \text { Men } & -0.07 & 219(122,324) & 219(122,324) & 0.703 \\ \text { All } & 0.169 & 183(81,324) & 183(81,32) & 0.263\end{array}$

Left ventricular end-systolic area

\begin{tabular}{lllll} 
Women & -0.086 & $34(24,52)$ & $34(30,38)$ & 0 \\
Men & -0.336 & $38(22,47)$ & $38(22,47)$ & -5.5 \\
All & -0.117 & $38(22,52)$ & $38(22,47)$ & -4 \\
Aortic cross-clamp time & & & & \\
Women & 0.163 & $122(51,219)$ & $122(51,187)$ & -0.125 \\
Men & -0.087 & $142(106,260)$ & $142(106,260)$ & 0.527 \\
All & 0.096 & $132(51,260)$ & $132(51,260)$ & 0.158 \\
\hline
\end{tabular}

Data reported as number (percent) patients, median (interquartile range), or ${ }^{\#}$ Spearman correlation coefficient, ${ }^{*} \mathrm{P}<0.05$; ${ }^{* * *} \mathrm{P}<0.01 ;{ }^{* * * *} \mathrm{P}<0.001$. 
There were 2 deaths within 30 days of surgery, including 1 woman $(2 \%)$ who died of multiple organ failure after primary aortic and mitral valve replacement and tricuspid valve repair, and 1 man $(5 \%)$ who died of aortic dissection after primary aortic and mitral valve replacement.

\section{Discussion}

This study showed that older age, atrial fibrillation, lower ejection fraction, and NYHA functional class II and III were associated with longer hospital and ICU stay in women. In men, longer cardiopulmonary bypass time was associated with greater left ventricular end-systolic area at 12 months after surgery. Other risk factors also may have influenced the outcomes of multiple valve procedures but could not be identified because of the small number of patients included in this study.

Several reports have assessed the effect of gender on isolated coronary artery bypass grafting procedures, with contradictory outcomes [1-6]. Women undergoing combined valvular surgery and coronary artery bypass grafting may be at greater risk of morbidity during the perioperative period, but long-term survival may be similar in woman and men [7]. The association between longer cardiopulmonary bypass duration and left ventricular end-diastolic area at 12 months in men may explain why men have higher levels of gene expression changes in response to cardiopulmonary bypass [8]. Direct comparison of outcomes between men and women may be confounded by differences in disease patterns and technical surgical considerations between men and women $[9,10]$. Nevertheless, women and men have similar benefits from combined procedures [7].

In summary, the present data suggest that 1) women and men have similar benefits from multiple valve procedures and 2) men experience more negative effects of prolonged cardiopulmonary bypass on left ventricular end-systolic area at 12 months after surgery than women.

\section{REFERENCES}

[1] F. H. Edwards, J. S. Carey, F. L. Grover, J. W. Bero and R. S. Hartz, "Impact of Gender on Coronary Bypass Operative Mortality," Annals of Thoracic Surgery, Vol. 66, No. 1, 1998, pp. 125-131. doi:10.1016/S0003-4975(98)00358-0
[2] V. Vaccarino, J. L. Abramson, E. Veledar and W. S Weintraub, "Sex Differences in Hospital Mortality after Coronary Artery Bypass Surgery: Evidence for a Higher Mortality in Younger Women," Circulation, Vol. 105, No. 10, 2002, pp. 1176-1181. doi:10.1161/hc1002.105133

[3] L. L. Mickleborough, S. Carson and J. Ivanov, "Gender Differences in Quality of Distal Vessels: Effect on Results of Coronary Artery Bypass Grafting," Journal of Thoracic and Cardiovascular Surgery, Vol. 126, No. 4, 2003, pp. 950-958. doi:10.1016/S0022-5223(02)73596-4

[4] C. G. Koch, F. Khandwala, N. Nussmeier and E. H. Blackstone, "Gender and Outcomes after Coronary Artery Bypass Grafting: A Propensity-Matched Comparison," Journal of Thoracic and Cardiovascular Surgery, Vol. 126, No. 6, 2003, pp. 2032-2043. doi:10.1016/S0022-5223(03)00950-4

[5] S. S. Khan, S. Nessim, R. Gray, L. S. Czer, A. Chaux and J. Matloff, "Increased Mortality of Women in Coronary Artery Bypass Surgery: Evidence for Referral Bias," Annals of Internal Medicine, Vol. 112, No. 8, 1990, pp. 561-567.

[6] O. Bech-Hanssen, I. Wallentin, E. Houltz, M. Beckman Suurküla, S. Larsson and K. Caidahl, "Gender Differences in Patients with Severe Aortic Stenosis: Impact on Preoperative Left Ventricular Geometry and Function, as Well as Early Postoperative Morbidity and Mortality," European Journal of Cardiothoracic Surgery, Vol. 15, No. 1, 1999, pp. 24-30. doi:10.1016/S1010-7940(98)00268-1

[7] T. Doenst, J. Ivanov, M. A. Borger, T. E. David and S. J. Brister, "Sex-Specific Long-Term Outcomes after Combined Valve and Coronary Artery Bypass," Annals of Thoracic Surgery, Vol. 81, No. 5, 2006, pp. 1632-1636. doi:10.1016/j.athoracsur.2005.11.052

[8] M. Ruel, C. Bianchi, T. A. Khan, S. Xu, J. R. Liddicoat, P. Voisine, E. Araujo, H. Lyon, I. S. Kohane, T. A. Libermann and F. W. Sellke, "Gene Expression Profile after Cardiopulmonary Bypass and Cardioplegic Arrest," Journal of Thoracic and Cardiovascular Surgery, Vol. 126, No. 5, 2003, pp. 1521-1530. doi:10.1016/S0022-5223(03)00969-3

[9] J. S. Lawton, S. J. Brister, K. R. Petro and M. Dullum, "Surgical Revascularization in Women: Unique Intraoperative Factors and Considerations," Journal of Thoracic and Cardiovascular Surgery, Vol. 126, No. 4, 2003, pp. 936-938. doi:10.1016/S0022-5223(03)00805-5

[10] C. G. Koch, C. M. Mangano, N. Schwann and V. Vaccarino, "Is It Gender, Methodology, or Something Else?" Journal of Thoracic and Cardiovascular Surgery, Vol. 126, No. 4, 2003, pp. 932-935. 